\title{
In Vitro-Transcribed Chrysanthemum stunt viroid RNA Is Infectious to Chrysanthemum and Other Plants
}

\author{
Yosuke Matsushita and Kumar K. R. Penmetcha
}

First author: Research Team for Growth and Flowering, National Institute of Floricultural Science, 2-1, Fujimoto, Tsukuba 305-8519, Japan; and second author: Functional Nucleic Acids, Institute for Biological Resources and Functions, National Institute of Advanced Industrial Science and Technology (AIST), Central 6, 1-1-1 Higashi, Tsukuba 305-8566, Japan. Accepted for publication 23 September 2008.

\begin{abstract}
Matsushita, Y., and Penmetcha, K. K. R. 2009. In vitro-transcribed Chrysanthemum stunt viroid RNA is infectious to chrysanthemum and other plants. Phytopathology 99:58-66.

Chrysanthemum stunt viroid (CSVd), a noncoding RNA, is known to cause chrysanthemum stunt disease, which affects the yield of flowers. To gain insights into CSVd replication, infection, and the reasons for the spreading of CSVd disease in chrysanthemum plants, we prepared linear

CSVd RNA and analyzed its ability to cause disease in chrysanthemum plants. We found that linear CSVd replicated as efficiently as CSVd RNA isolated from the infected chrysanthemum plants. Additionally, the linear CSVd RNA was evaluated for its ability to infect other plants as well, which revealed that CSVd has a wide host range for its replication. Importantly, the CSVd isolated from these hosts is infectious to chrysanthemum plants, and thus potentially contributes to the spreading of the disease to chrysanthemum plants.
\end{abstract}

Viroids are the smallest, self-replicating, noncoding RNAs capable of inducing a full-range of disease symptoms in susceptible host plants, including potato, tomato, cucumber, hop, coconut, grapevine, fruit trees (avocado, peach, apple, pear, citrus, and plum), and some flowering plants (chrysanthemum and coleus) (5). The viroids are single-stranded RNAs, which range from 250 to 400 nucleotides (nts) in length, and they exist as a circular structure with a high degree of self-complementarity, either to promote compact folding or to elicit their function(s). It is remarkable that the short genomes of the viroids are able to contain all of the information related to intracellular trafficking, localization, replication, and pathogenicity. Since the discovery of Potato spindle tuber viroid (PSTVd [3]) over 3 decades ago, a number of viroids have been reported. Currently, about 30 species of viroids and their variants are known (5). All viroids fall into two distinct families: Pospiviroidae and Avsunviroidae. In Pospiviroidae viroids, the secondary structure of the RNA is either quasi-double-stranded or rodlike, whereas the RNA in Avsunviroidae viroids assumes highly branched secondary structures. These viroids are clearly distinguished by other properties as well, such as the presence or absence of structural and functional motifs, and the mode of replication.

Previous work on PSTVd suggested that the sequence difference between the severe and mild strains is located between the left terminal domain and the central conserved region $(8,11)$. These studies led to the proposal of five structural domains (leftterminal domain, pathogenicity domain, central domain [containing the central conserved region], variable domain, and right-terminal domain) in the secondary structure of the viroids in Pospi-

Corresponding author: K. K. R. Penmetcha; E-mail address: pkr-kumar@aist.go.jp

* The $\boldsymbol{e}$-Xtra logo stands for "electronic extra" and indicates that the online version contains three additional figures showing analysis of CSVd infection using RT-PCR analysis and comparison of primary CSVd RNA sequences of CSVd isolated from infected chrysanthemum, petunia, and potato plants.

doi:10.1094/PHYTO-99-1-0058

(C) 2009 The American Phytopathological Society viroidae. However, subsequent studies revealed that the correlation between the structural elements of viroids and the severity of symptoms appeared to be too complex to assign specific domains to the pathogenicity $(17,21)$.

In order to identify the important sequences in viroids for pathogenicity, replication, and systemic RNA trafficking studies, RNA isolated from infected plants, infectious cDNA clones and in vitro-transcribed RNAs have been widely used $(2,4,9,15-$ 19,23,24,26). In the past, in vitro-transcribed linear RNAs (+) of both PSTVd and Citrus exocortis viroid (CEVd) were shown to be infectious to their cognate plants (17-20,24). Recently, variants of PSTVd were evaluated for vascular entry specifically from the bundle sheath into the phloem. This study not only identified an RNA tertiary structural motif responsible for trafficking but also suggested the importance of combining different analyses, such as 3D motif searches, comparisons with $\mathrm{X}$-ray crystal structures, mutagenesis and covariations, to evaluate the functions of the RNA motifs in the viroids (28).

Chrysanthemum stunt viroid (CSVd) was first recognized in the United States in 1945, and by the 1950s, the disease had spread rapidly all over the world, affecting both the plants and flowers of different chrysanthemum (Chrysanthemum morifolium Ramat.) cultivars. The widespread nature of CSVd disease in chrysanthemum plants and the potential reasons for the spreading of the disease are intriguing research topics. CSVd was previously shown to replicate in a wide host range of plants, such as the families Solanaceae, Asteraceae, and Cucurbitaceae (1). It is possible that some of these plants may also contribute to the spread of CSVd disease in chrysanthemum plants. Importantly, most of the cultivated chrysanthemum (C. morifolium) plants display CSVd disease symptoms, such as stunting of plant height, reduced flower size, and flower color bleaching (10), whereas no such symptoms are observed in other plants, including wild-type chrysanthemum (C. pacificum Nakai) plants (13).

In spite of the economic losses endured by floriculturists due to CSVd disease, few studies on CSVd infection and replication have been done, and are partly hampered by the absence of in vitro infectious clones. In the present study, to address this issue, we prepared a linear CSVd RNA by in vitro transcription and 
found that it could infect chrysanthemum plants. This CSVd RNA appeared to replicate in chrysanthemum plants as efficiently as the CSVd RNA isolated from infected plants, suggesting that both the linear and circular forms of CSVd are competent for infection. The transcribed CSVd RNA was able to replicate in plants belonging to the families Solanaceae and Asteraceae, without inducing any morphological symptoms, which are similar results to those obtained using extracts of CSVd-infected plants (1). The CSVd RNA isolated from these plants retained its ability to cause infection in CSVd-free chrysanthemum plants. Due to this property, we believe that other host plants may serve as reservoirs for transmitting the CSVd disease to the chrysanthemum plants, and thus may explain the reasons why CSVd disease is widespread in chrysanthemum plants.

\section{MATERIALS AND METHODS}

Isolation of CSVd RNA from infected chrysanthemum plants. CSVd-infected and uninfected control chrysanthemum $(C$. morifolium) plants were propagated in a greenhouse at 20 to $30^{\circ} \mathrm{C}$ under a long photoperiod $(14 \mathrm{~h})$ to prevent flowering. Total RNA was extracted from $20 \mathrm{~g}$ of frozen $\left(-20^{\circ} \mathrm{C}\right)$ chrysanthemum leaves of infected and uninfected plants using TRIzol (Invitrogen), according to the manufacturer's instructions. Briefly, the RNAs were separated with $2 \mathrm{M} \mathrm{LiCl}$ for $2 \mathrm{~h}$ at $4^{\circ} \mathrm{C}$ (22). Ethanol was added to the supernatants to a final concentration of $35 \%$ ( $\mathrm{vol} / \mathrm{vol}$ ), and then the solution was passed through the SV total RNA isolation system column (Promega). The column was washed with $35 \%$ ethanol ( $\mathrm{vol} / \mathrm{vol})$ several times, and then was eluted with distilled water. The RNA was then fractionated by $15 \%$ native polyacrylamide gel electrophoresis (PAGE). The separated bands were visualized under ultraviolet light after staining with ethidium bromide. The CSVd RNA (less than 500 base pairs) was clearly visible on the gel in the RNA isolated from the infected plants (Fig. 1A). This RNA was excised from the gel and incubated overnight in $2 \times$ STE buffer $(100 \mathrm{mM}$ Tris- $\mathrm{HCl}, 200 \mathrm{mM} \mathrm{NaCl}$, and $2 \mathrm{mM}$ EDTA, pH 7.2) at room temperature. The gel slurries were then pelleted by low-speed centrifugation, 3,000 $\times g$, and the supernatants were collected. To confirm that the isolated RNA was CSVd, we also performed a reverse transcription-polymerase chain reaction (RT-PCR) analysis, as described (13) using the CSVd-specific primers, CSVd-r and CSVd-f (Table 1). The resulting amplified PCR product was $253 \mathrm{bp}$ long.

Cloning and sequence analysis of the isolated CSVd. To determine the sequence of the isolated CSVd RNA, we cloned the above isolated PCR product into the pGEM-T Easy vector (Promega) and sequenced the entire insert, using a BigDye Terminator cycle sequencing kit (Applied Biosystems) with M13 primer sets.

In vitro synthesis of CSVd RNA. To prepare both the CSVd (+) and CSVd (-) RNAs, we initially amplified the PCR product using a vector containing the CSVd sequence (pT7-CSVd) with two sets of primers, CSVd-R and CSVd-F, CSVd-mR and CSVd$\mathrm{mF}$, to prepare the templates for generating (+) and (-) RNAs, respectively (Table 1$)$. The resulting PCR products were used as templates for in vitro transcription, which was performed at $37^{\circ} \mathrm{C}$ for about $12 \mathrm{~h}$ with an Ampliscribe T7 kit (Epicentre Technologies). After treatment with DNase I, the reaction mixtures were fractionated on an $8 \%$ native polyacrylamide gel. The RNAs were extracted from the gel, and were quantified as described (,7). The CSVd (+) and CSVd (-) RNAs were both tested for their ability to infect the chrysanthemum plants, along with the PCR product, the plasmid (pT7-CSV), and the RNA isolated from the infected plants.

CSVd infection analysis in plants. To analyze CSVd replication in plants with the prepared nucleic acids (plasmid [pT7CSV], PCR product, transcribed RNAs), we used the wild-type chrysanthemum, C. pacificum, as it is known for its sensitivity to
CSVd infection. Each chrysanthemum plant was inoculated with $2.5 \mu \mathrm{g}$ of CSVd (+) or CSVd (-) RNA or PCR product or plasmid. The inoculation was carried out by needle puncture of the stem (Fig. 1A). As a positive control, total RNA extracted from CSVdinfected chrysanthemums was used, and as a negative control, inoculations were performed with nuclease-free water. At least six plants were used for testing each nucleic acid sample for the initial experiments, whereas three plants were used to test the infectivity of CSVd on different plant species. The inoculated plants were allowed to grow at 20 to $24^{\circ} \mathrm{C}$ under a long photoperiod. At 2 weeks and beyond postinoculation, the expanded leaves above the inoculation site were collected, and the isolated total RNA was subjected to RT-PCR analysis, as described above (Fig. 1B). To analyze the CSVd replication in other plants, we performed a bioassay similar to that as described above, by inoculating $2.5 \mu \mathrm{g}$ of CSVd nucleic acids per plant into ageratum (Ageratum houstonianum Mill.), Garland chrysanthemum $(C$. coronarium L.), and tomato (Solanum lycopersicum L.).

Isolation, cloning and sequencing of CSVd isolated from other plants. Total RNA was extracted, as described above, from the infected tomato and ageratum plants, and RT-PCR was performed using two sets of sequencing primers (CSVd-94-f and CSVd-94-r). The RT-PCR conditions were similar to those described previously (13). The resulting PCR product (full-length CSVd) was cloned into the pGEM-T Easy vector and the entire insert was sequenced, using a BigDye terminator cycle sequencing kit (Applied Biosystems) with M13 primer sets.

CSVd isolated from other plants to re-infect chrysanthemum plants. We performed re-infection studies on CSVd RNAs isolated from ageratum and tomato plants, to infect wild-type chrysanthemum (C. pacificum), ageratum, and tomato plants. Infection studies were carried out by needle injection, as mentioned above. For inoculating RNA into fresh tomato plants, we used 9.6 and $2.4 \mu \mathrm{g}$ of total RNA, isolated from infected tomato and ageratum plants, respectively. For inoculating RNA into uninfected wild-type chrysanthemum plants, we used 9.6 and $2.4 \mu \mathrm{g}$ of total RNA, isolated from infected tomato and ageratum plants, respectively. For inoculating RNA into fresh ageratum plants, we used 30.2 and $16.5 \mu \mathrm{g}$ of total RNA, isolated from infected tomato and ageratum plants, respectively. In the latter case we used more RNA, since no infection was observed with 9.6 and $2.4 \mu \mathrm{g}$ of total RNA.

Grafting assays. Eight-week-old wild-type chrysanthemum (C. pacificum) plants, which were inoculated with transcribed CSVd RNA, were cut, and the roots were used for grafting. Scions of uninfected cultivated chrysanthemum (C. morifolium) plants were grafted onto the infected plant roots of the wild-type chrysanthemum plants, as previously described (Fig. 2A). Grafted plants were kept for 8 weeks at room temperature $\left(20\right.$ to $\left.24^{\circ} \mathrm{C}\right)$. Total RNA was extracted from the scion leaves of the 8-week-old grafted plants and was analyzed by RT-PCR, as described above. To compare the heights of the infected cultivated chrysanthemum plants with those of the uninfected cultivated chrysanthemum plants, we collected the scions from these plants, replanted and allowed them to grow for about 4 months before comparing the length of the plants (Fig. 2B). Similarly, we also carried out grafting assays using scions of petunia (Petunia hybrida Vilm.) and potato (Solanum tuberosum L.) plants. The petunia and potato grafted plants were kept for 12 and 16 weeks, respectively, at room temperature before the infection was analyzed by RT-PCR. The scions from these infected petunia and potato plants were replanted and allowed to grow for about one year. To analyze the CSVd-RNA sequences in these infected plants, we collected the upper leaves of the scions, isolated the total RNA, amplified it by RT-PCR, cloned the fragment into the pGEM-T Easy vector (Promega), and sequenced the entire insert, as described above. 
A
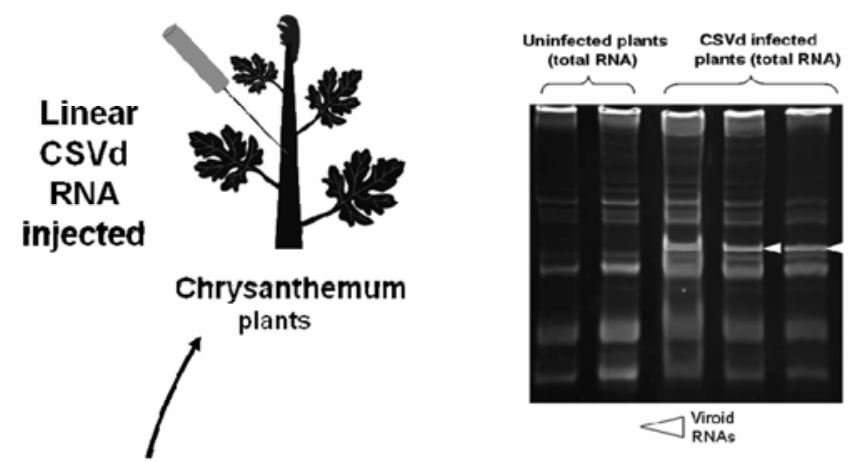

Isolated RNA

from the gel

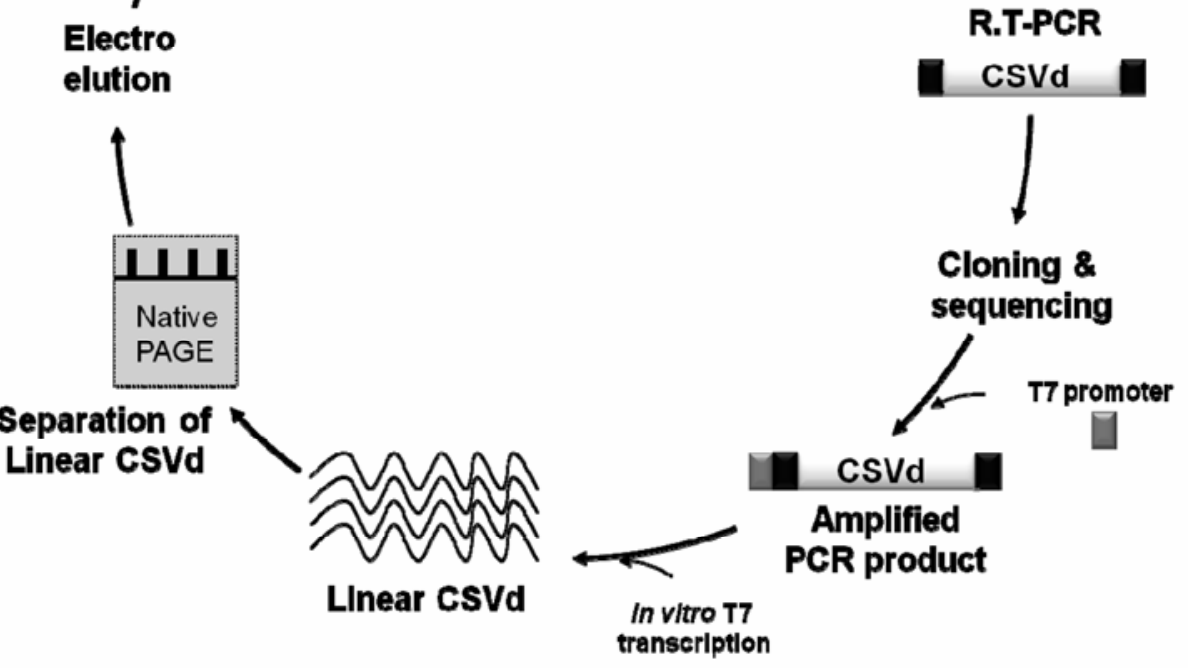

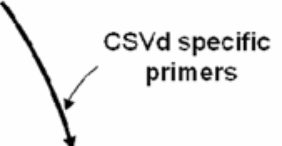

R.T-PCR

csvd

\section{Cloning \&}

sequencing

CSVd

Amplified

In vitro T7

transcription

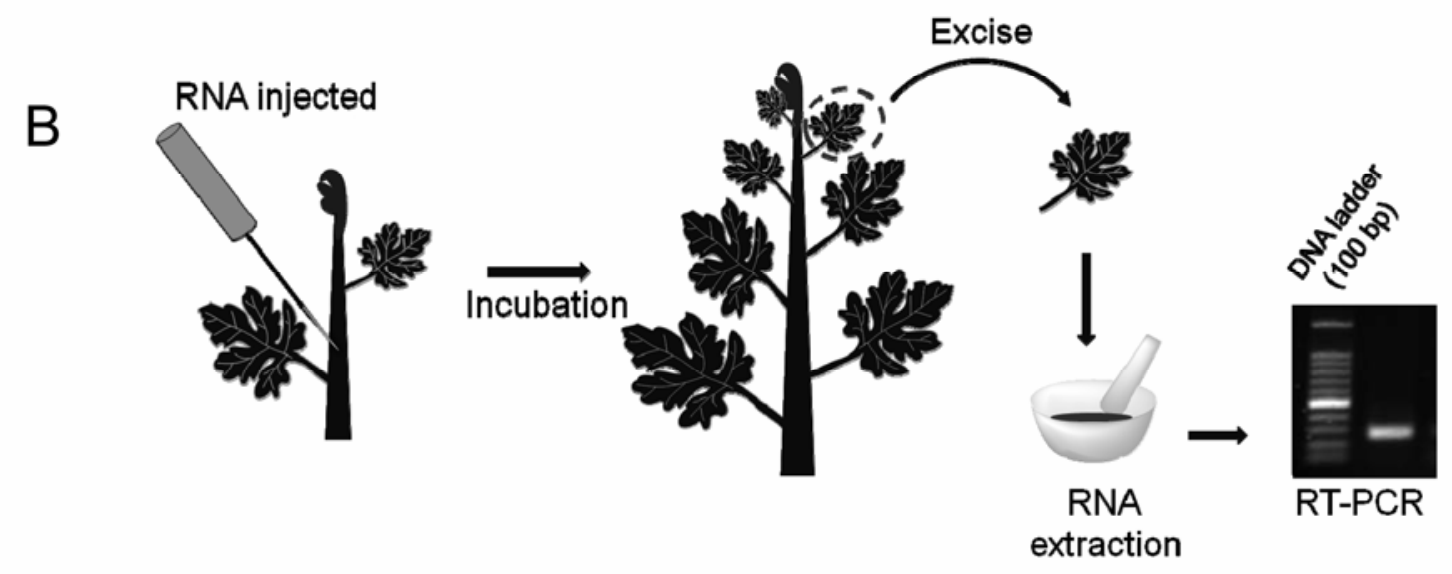

C

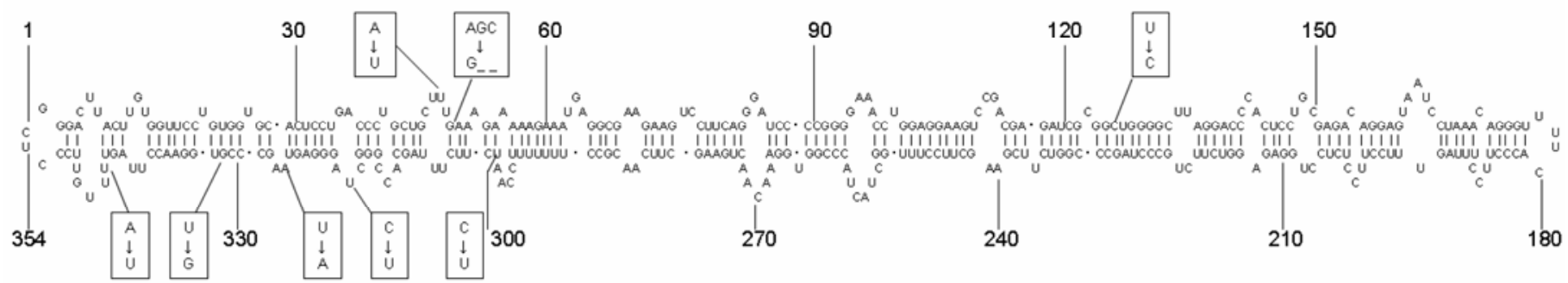

Fig. 1. Isolation, identification and in vitro synthesis of Chrysanthemum stunt viroid (CSVd) RNA. A, Isolation of CSVd RNA from the infected chrysanthemum plants and in vitro synthesis of CSVd RNA. B, Analysis of CSVd replication after inoculation of CSVd nucleic acids. C, Complete sequence and proposed secondary structure of isolated CSVd (X16408). The nucleotide substitutions and deletions from CSVd (V01107, [9]) are shown within boxes. The nucleotides at positions 78 to 105 and 255 to 280 are the conserved regions in the genus Pospiviroidae. 


\section{RESULTS AND DISCUSSION}

Preparation of CSVd RNA. To identify the sequence of the CSVd strain from the infected cultivated chrysanthemum $(C$. morifolium) plants, we isolated total RNA from both infected and uninfected chrysanthemum plants, and analyzed it by PAGE under nondenaturing conditions. A comparison of the RNA bands from the two samples on the gel revealed a distinct band (indicated by the arrow in Fig. 1A). This RNA band was observed only in the RNA isolated from infected chrysanthemum plants, and the band migrated at about $400 \mathrm{nts}$. To determine whether the isolated RNA was CSVd, we performed an RT-PCR analysis using specific primers for CSVd. A clear, distinct PCR product, representing about 360 nts, was observed (data not shown). These analyses clearly suggested that the isolated RNA was, indeed, CSVd. Next, to determine the sequence of the isolated RNA, we sequenced the entire insert after cloning the RT-PCR product into a vector (pT7-CSV). We sequenced five clones, and found that all of them had the same sequence, as shown in Figure 1C. This isolated strain is the major CSVd strain circulating in Japan and infecting chrysanthemum plants in the field (13), as previously reported (GenBank accession no. X16408). We compared our isolated CSVd sequence with that of the first CSVd reported more than 2 decades ago (9), and found a few substitutions in the RNA sequence, as indicated in the boxes adjacent to the sequence. These variations in the primary sequences of CSVd could have originated from the RNA-dependent RNA polymerase in chrysanthemum plants, which lacks proofreading ability.

CSVd RNA replication in wild-type chrysanthemum plants. In vitro-transcribed RNAs have been widely used for CEVd and PSTVd in order to identify the important sequences in viroids for pathogenicity, replication, and systemic RNA trafficking studies. However, no information is available, at present, about the replication ability of in vitro-transcribed linear CSVd RNAs in chrysanthemum plants, which has hampered the progress in the evaluation of important CSVd sequences for infection, replication and pathogenicity. For the above reasons, we prepared both (+) and (-) RNAs of CSVd by in vitro transcription, and tested their ability to replicate in chrysanthemum (C. pacificum) plants. In addition to these samples, we also prepared two other products, the PCR product and the plasmid (pT7-CSV), to test their ability to replicate in plants. These four nucleic acid preparations were then each injected by needle puncture of the stem into uninfected chrysanthemum plants, which were incubated for at least 2 weeks prior to their evaluation by RT-PCR analysis. As shown in Table 2, only the CSVd (+) RNA replicated with efficiency similar to that of the RNAs isolated from the infected chrysanthemum plants. The presence of CSVd RNA was detectable at 3 weeks postinoculation, in both the transcribed CSVd and the CSVd isolated from the infected chrysanthemum plants. By the end of 5 weeks, CSVd was detected in almost all of the plants inoculated with these two RNAs. These studies suggested that the linear CSVd RNA has the potential to replicate and to be transported efficiently in the host plants. Previously, isolated circular CSVd RNAs (from infected chrysanthemum plants), which were linearized by $\mathrm{Mg}^{2+}$ ion-mediated cleavage, were shown to retain the ability to infect Gynura aurantiaca plants (16), which belong to the chrysanthemum family. However, in that study, it was difficult to establish whether all of the isolated circular CSVd RNAs were converted to the linear form upon cleavage with $\mathrm{Mg}^{2+}$ ions. Our present results unambiguously show for the first time that unit-length linear CSVd (+) RNA has the ability to replicate in chrysanthemum plants. However, CSVd (-) RNA failed to infect $C$. pacificum plants. This could be due to sensitivity toward ribonucleases, inefficient replication of the (-) sense RNA or lack of recognition by the proteins that are involved in transportation and localization of (+) RNA, as suggested for PSTVd (24). We also tested the dimeric form of CSVd, and found that it has similar infectious ability as well (data not shown).

Transcribed CSVd RNA reproduces disease symptoms in chrysanthemum plants. The above studies suggested that the in vitro-transcribed CSVd RNA is able to replicate efficiently in chrysanthemum (C. pacificum) plants. However, concerns regarding RT-PCR methods as exclusive proof for viroid infection have been raised, because past experiences have shown that amplicon contamination is not infrequent, and it can lead to erroneous conclusions. It is thus important to reproduce clear-cut symptoms in cultivated chrysanthemum (C. morifolium) using transcribed CSVd RNA, as clear proof of infectivity. Therefore, we used a grafting assay, as described in detail in the materials and methods, since the transmission of CSVd was found to be straightforward and reproducible (Fig. 2A; 1). As shown in Figure 2B, the infected plant clearly showed stunting, a typical symptom of CSVd, compared with the uninfected plant, which is in agreement with the RT-PCR analysis. Taken together, these analyses suggest that the transcribed CSVd RNA, has the ability to replicate and cause CSVd disease in the cultivated chrysanthemum plants.

CSVd RNA replication in other plants. The sap from CSVdinfected chrysanthemum plants is able to infect many plants, and thus CSVd is known to have a wide host range for its replication (1). To address whether the in vitro-transcribed RNA also retained this ability to infect other plants, we tested three plants. Among them, two belonged to the same family as chrysanthemum $(A$. houstonianum and C. coronarium), and one plant, S. lycopersicum, was from the family Solanaceae. We inoculated the transcribed CSVd (+) RNA into these plants, as described above. At 3 weeks postinoculation, we carried out RT-PCR analyses to detect the CSVd RNA in these plants, after isolating the total RNA from the plant leaves. Interestingly, all of these plants showed the presence of replicated CSVd RNA (Table 3), suggesting that the transcribed RNA efficiently replicated in these plants. In the ageratum and tomato plants, the replicated RNA was detected at 4 weeks postinoculation, while in the $C$. coronarium plant, we observed it at 6 weeks postinoculation, suggesting that the CSVd RNA replication efficiency may vary, depending on the host plant, and even among plants belonging to the same family (Table 3 ). Previously, the isolated CSVd RNA was shown to infect ageratum

TABLE 1. Oligonucleotide primers used for in vitro synthesis of Chrysanthemum stunt viroid (CSVd) RNA and RT-PCR ${ }^{\mathrm{a}}$

\begin{tabular}{lllr}
\hline \multicolumn{1}{c}{ Method } & \multicolumn{1}{c}{ Sequence $\left(5^{\prime}-3^{\prime}\right)$} & $\begin{array}{c}\text { Target of amplification } \\
\text { (position number) }\end{array}$ & \multicolumn{1}{c}{} \\
\hline CSVd-R & In vitro transcription (+RNA) & GAGGGAACAAAACTAAGGTTCCACGGGC & $328-1$ \\
CSVd-F & In vitro transcription (+RNA) & AGAGTAATACGACTCACTATAGGGACTTACTTGTGGTTCCTGTGG & $2-25$ \\
CSVd-mR & In vitro transcription (-RNA) & CGGGACTTACTTGTGGTTCCT & $1-21$ \\
CSV-mF & In vitro transcription (-RNA) & AGAGTAATACGACTCACTATAGGGAACAAAACTAAGGT & $337-354$ \\
CSVd-r & RT-PCR & AGGATTACTCCTGTCTCGCA & $148-167$ \\
CSVd-f & RT-PCR & CAACTGAAGCTTCAACGCCTT & $269-290$ \\
CSVd-94-r & Sequence & GGATCCCTGAAGGACT & $88-74$ \\
CSVd-94-f & Sequence & CCGGGGAAACCTCCAG & $90-105$ \\
\hline
\end{tabular}

a The T7 RNA polymerase promoter sequence is depicted in bold letters. 
(1) and tomato (14), after mechanical inoculation for analyzing the infection and disease. Taken together, these studies suggested that the transcribed CSVd RNA as well as the sap of the CSVd infected plants can replicate and infect the families Asteraceae and Solanaceae. However, no disease symptoms were visible in these plants, except in the chrysanthemum plants.

Next, we were interested in determining whether the replicated RNA retains its primary sequence or undergoes base substitutions or deletions. For this, we amplified, cloned and sequenced about 5 to $10 \mathrm{CSVd}$ clones from each infected ageratum and tomato plant and compared the primary sequences. No substitutions or deletions in the primary sequence of CSVd were observed in the CSVd isolated from the ageratum and C. coronarium strains (Fig. 3). Additionally, we have carried out a second round of infection in Ageratum plants after isolating the CSVd (X16408) from the above infected plants and no substitutions of bases were observed in CSVd sequences (among five clones). However, a few substitutions, predominantly transitions (purine substituted by purine and pyrimidine substituted by pyrimidine), were observed when the inoculated RNA was analyzed from the infected tomato and wild-type chrysanthemum (C. pacificum) plants. These sequence analyses of the CSVd isolated from these two plants suggested that the CSVd strains may undergo transitions, depending upon the host plants.
CSVd RNA isolated from other plants is infectious to chrysanthemum plants. It is important to determine whether the replicated CSVd RNA isolated from the ageratum and tomato plants (with the transition substitutions) is infectious to chrysanthemum plants. Therefore, we isolated total RNA from these plants and used it to inoculate uninfected wild-type chrysanthemum, tomato and ageratum plants. After 4 weeks, we analyzed the plants for the presence of CSVd RNA. As shown in Table 4, the isolated CSVd RNAs from the tomato and ageratum plants were able to infect both chrysanthemum and tomato plants, whereas the ageratum plant was only infected when the CSVd was isolated from

TABLE 2. Ability of Chrysanthemum stunt viroid (CSVd) nucleic acids to replicate in chrysanthemum

\begin{tabular}{lcc}
\hline & \multicolumn{2}{c}{ Postinoculation (week) $^{\mathrm{a}}$} \\
\cline { 2 - 3 } Inoculum & 4 & 5 \\
\hline Plasmid & $0 / 6$ & $0 / 6$ \\
cDNA & $0 / 6$ & $0 / 6$ \\
(-) RNA transcripts & $0 / 6$ & $0 / 6$ \\
(+) RNA transcripts & $3 / 6$ & $5 / 6$ \\
CSVd RNA & $6 / 6$ & $6 / 6$ \\
mock & $0 / 6$ & $0 / 6$ \\
\hline
\end{tabular}

a Number of CSVd replication plants/number of plants inoculated.
A

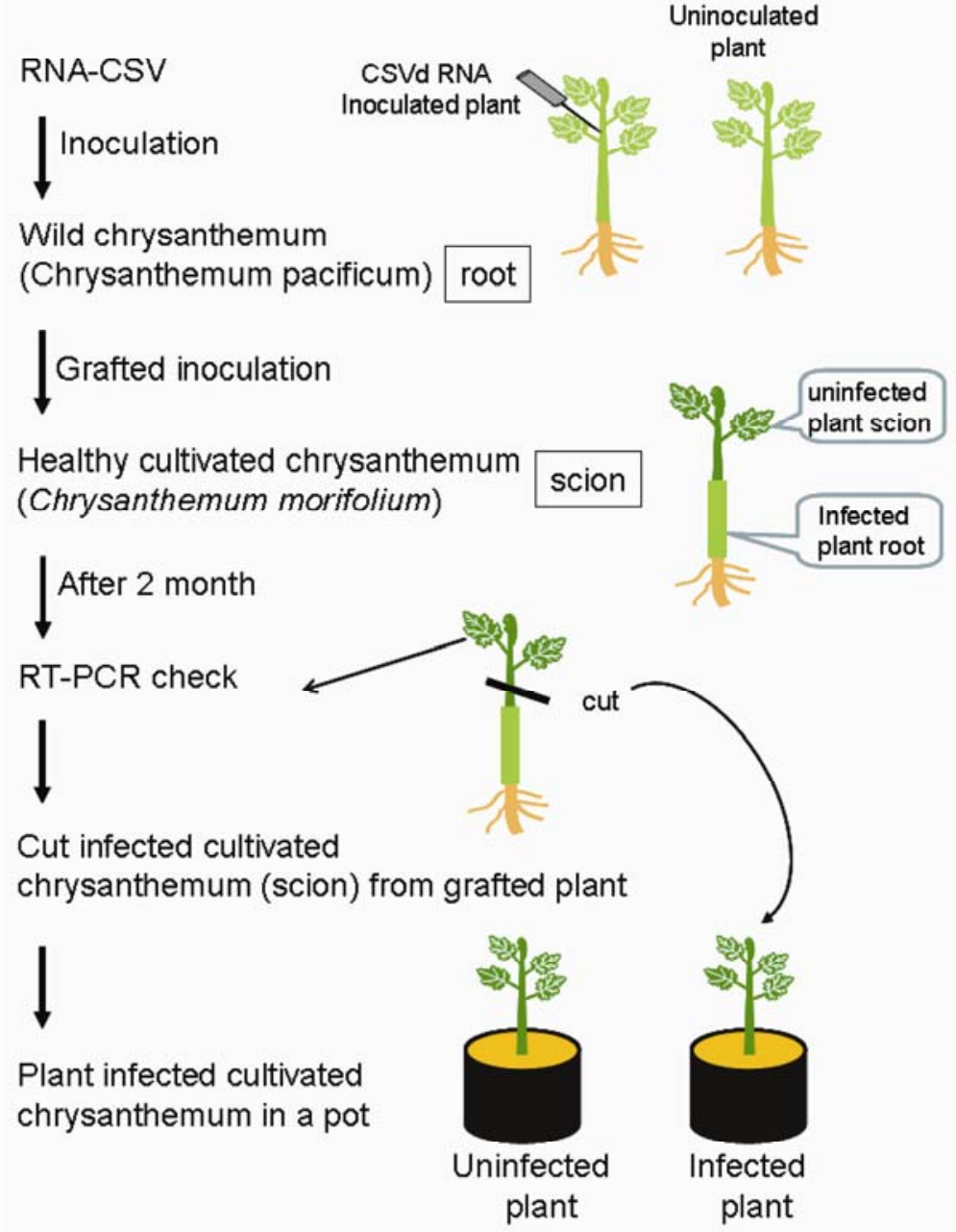

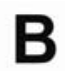

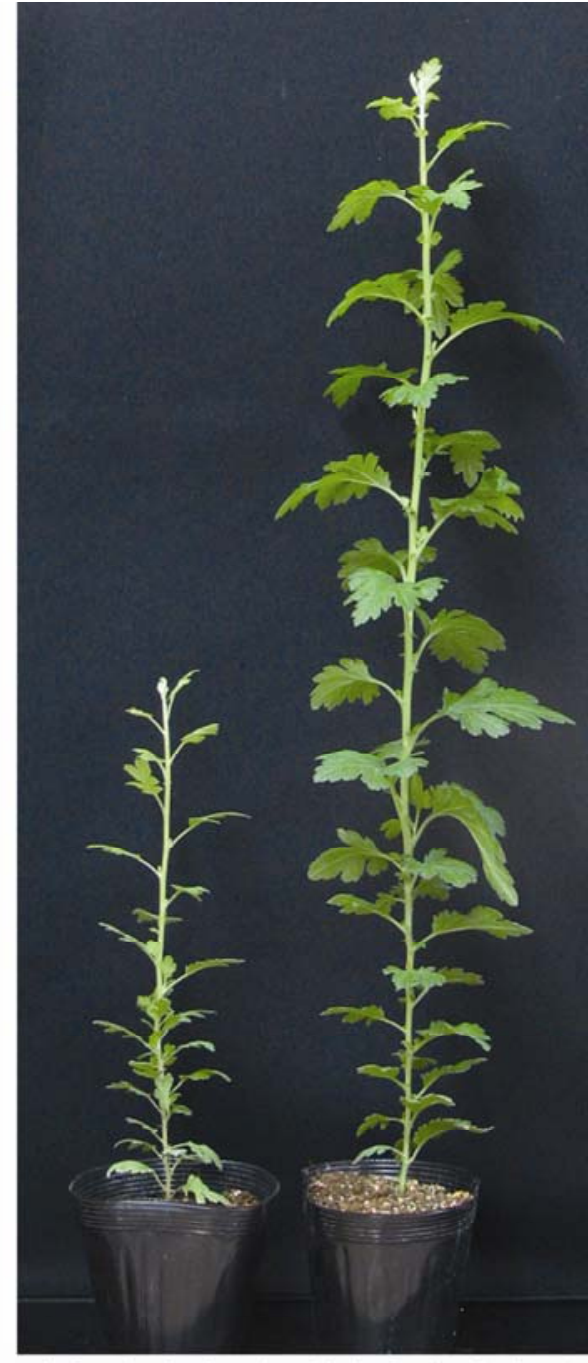

Infected plant Uninfected plant

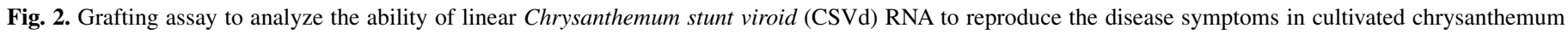

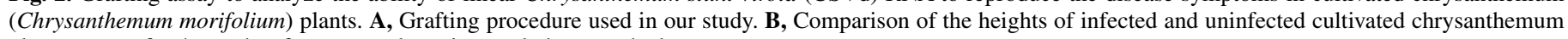
plants, grown for 4 months after retransplantation, and photographed. 
either the same species or the chrysanthemum plants, but not from the other plants. These variations in the CSVd infectivity might have originated from the CSVd substitutions observed above in the CSVd infected tomato plants. In contrast, the chrysanthemum plants became infected with CSVd when the inocula from the tomato and ageratum plants were used. These studies clearly suggested that the CSVd is not only able to replicate efficiently in a wide range of plants but also retains its ability to infect new chrysanthemum plants. The reason for this could be due to the absence of host-specific CSVd sequence requirements in chrysanthemum plants. Thus, it appears that while CSVd RNA is passing through different hosts, it undergoes variations in its primary sequence (Fig. 3), and by this process newer viroids may evolve, which may have better adaptability to other plants or cause severe forms of CSVd. This kind of host-driven evolution brought on by the host polymerase appears to be advantageous to the viroids.

In view of the above proposal, we performed additional studies to determine if the CSVd-infected tomato plants can transmit the viroids to other plants and establish host-specific infectious CSVd RNA. For this, we grafted petunia and potato plants or twigs with CSVd-infected tomato plants (infected with the CSVd X16408 strain) for 3 or 4 months, to facilitate the production of CSVd variants to establish infectivity into these two hosts (Figs. 4 and

TABLE 3. Ability of linear Chrysanthemum stunt viroid (CSVd) (+) RNA to replicate in other plants

\begin{tabular}{|c|c|c|c|c|c|}
\hline \multirow[b]{2}{*}{ Family } & \multirow[b]{2}{*}{ Inoculated plants } & \multirow[b]{2}{*}{ Inoculum } & \multicolumn{3}{|c|}{ Postinoculation (week) ${ }^{\mathrm{a}}$} \\
\hline & & & 4 & 6 & 8 \\
\hline \multirow[t]{6}{*}{$\overline{\text { Asteraceae }}$} & Chrysanthemum pacificum & (+) RNA & $3 / 6$ & $5 / 6$ & $5 / 6$ \\
\hline & & Mock & $0 / 6$ & $0 / 6$ & $0 / 6$ \\
\hline & Ageratum houstonianum & (+) RNA & $7 / 10$ & $9 / 10$ & $9 / 10$ \\
\hline & & Mock & $0 / 3$ & $0 / 3$ & $0 / 3$ \\
\hline & Chrysanthemum coronarium & (+) RNA & $0 / 8$ & $4 / 8$ & $7 / 8$ \\
\hline & & Mock & $0 / 3$ & $0 / 3$ & $0 / 3$ \\
\hline \multirow[t]{2}{*}{ Solanaceae } & Solanum lycopersicum & (+) RNA & $6 / 10$ & $8 / 10$ & $8 / 10$ \\
\hline & & Mock & $0 / 4$ & $0 / 4$ & $0 / 4$ \\
\hline
\end{tabular}

a Number of CSVd replication plants/number of plants inoculated.

\section{A CSVd isolated from ageratum and Chrysanthemum coronarium plants (All 8 clones)}

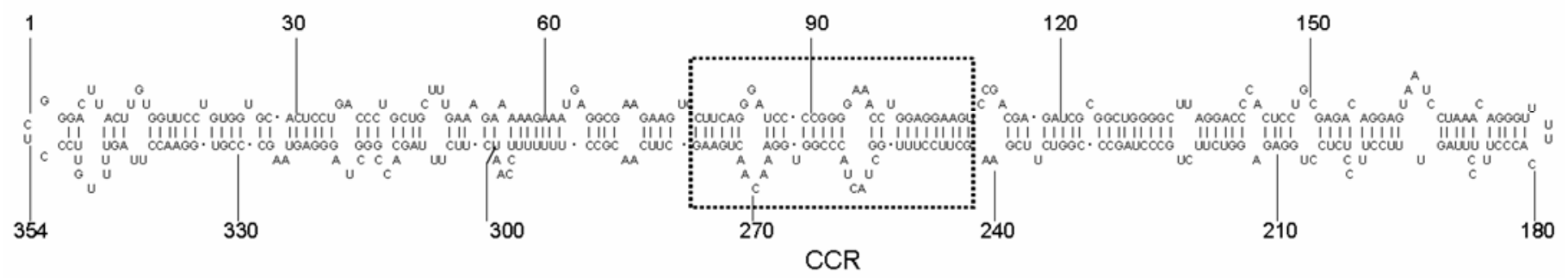

\section{B CSVd isolated from tomato plants}

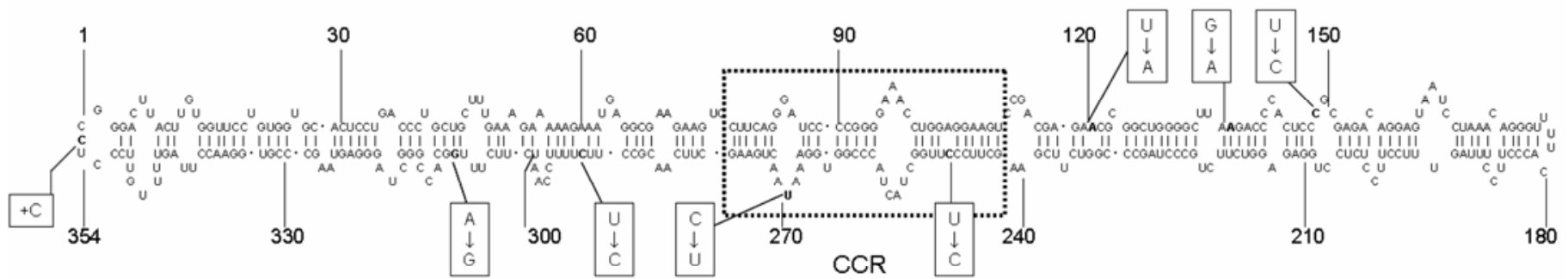

\section{CSVd isolated from wild-type chrysanthemum plants}

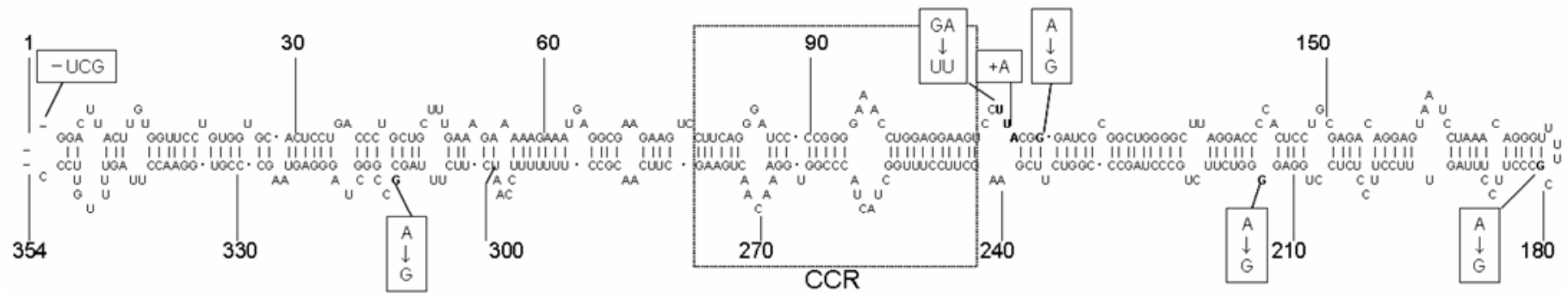

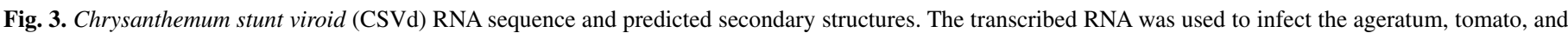

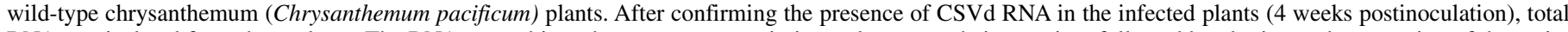

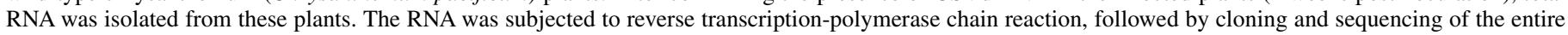

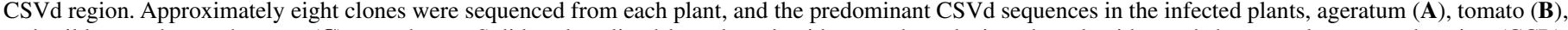

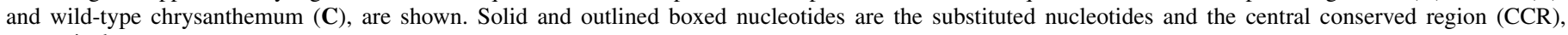
respectively. 
5). The infected petunia and potato scions were each cut and grown additionally for about 1 year before the isolation of CSVd RNA from these plants. The RNA was amplified by RT-PCR, cloned into the pGEM-T Easy vector (Promega), and sequenced in its entirety. We sequenced eight and five clones from petuniaand potato-derived CSVd, respectively. All of the petunia-derived CSVd clones (referred to as CSVd-Petu) have a single G residue insertion after nucleotide 130, as well as three base substitutions (Fig. 4). In order to understand the importance of the $G$ residue insertion and the substitutions, we compared the petunia-derived CSVd sequences reported here with that of the previously reported natural isolate of CSVd, isolated from naturally infected petunia plants (25). We found that the $G$ base insertion was common in both the natural isolate and our infectious CSVd RNA, which suggests that the insertion of a $G$ residue is essential for establishing CSVd infection in petunia. Additionally, we confirmed the requirement of $\mathrm{G}$ base insertion to establish CSVd infection in petunia by second round of infection into the petunia plants, followed by isolation and sequence of the replicated CSVd in petunia. We observed that among five sequenced clones all retained the $G$ insertion and no other changes were noticed. On the other hand, the potato-derived CSVd (referred as CSVd-Pota) had base substitutions at the 47th and 49th positions among all five of the sequenced clones. These insertions and substitutions in CSVd appear to alter the secondary structures of the CSVd RNA

TABLE 4. Ability of Chrysanthemum stunt viroid (CSVd) (+) RNA isolated from other plants to infect either chrysanthemum plants or other plants

\begin{tabular}{llc}
\hline Inoculated plants & \multicolumn{1}{c}{ Inoculum } & $\begin{array}{c}\text { Infectivity } \\
\text { (infected plants/test plants) }\end{array}$ \\
\hline Chrysanthemum pacificum & Tomato CSVd & $2 / 4$ \\
& Ageratum CSVd & $2 / 4$ \\
Solanum lycopersicum & Tomato CSVd & $6 / 7$ \\
\multirow{2}{*}{ Ageratum houstonianum } & Ageratum CSVd & $2 / 3$ \\
& Tomato CSVd & $0 / 4$ \\
& Ageratum CSVd & $3 / 3$ \\
\hline
\end{tabular}

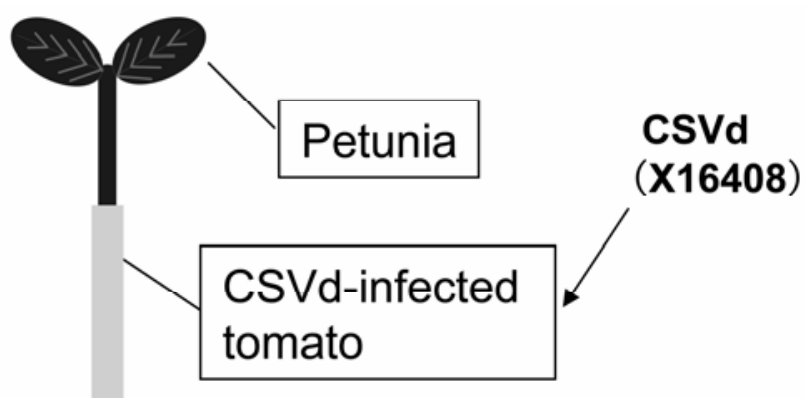

Incubation
for 3 months
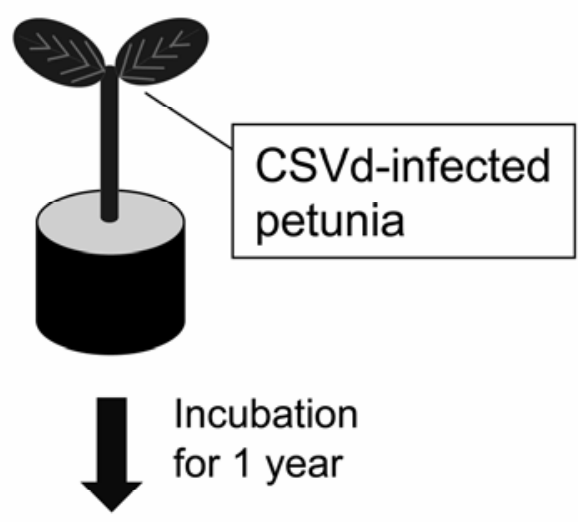

RT-PCR and Sequencing

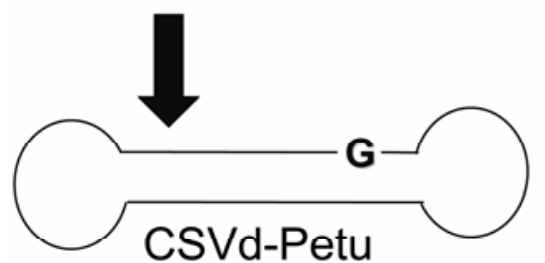

(Sequenced 8 clones)

\section{Predicted of secondary structure of CSVd near the mutation site}

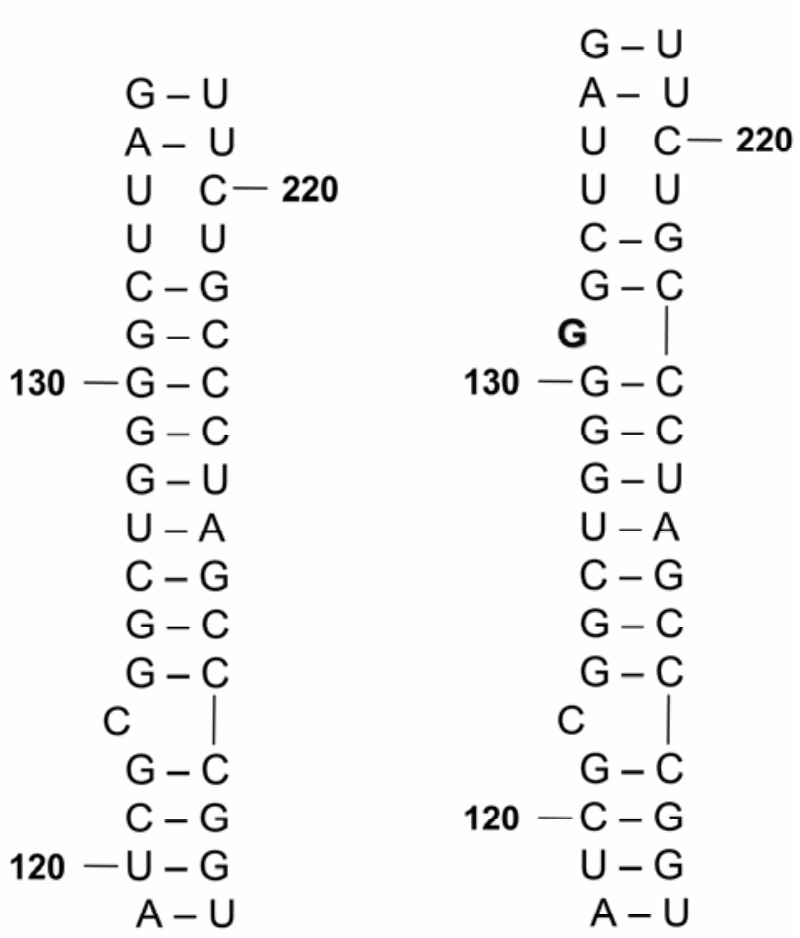

CSV-X16408

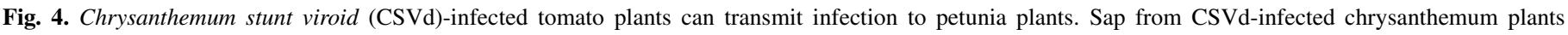

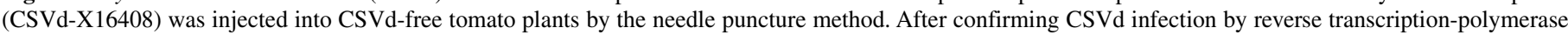

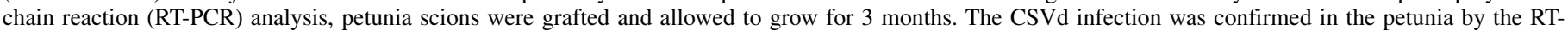

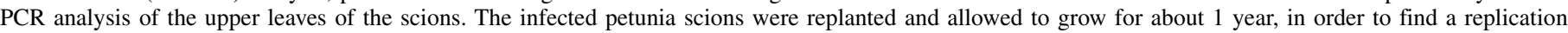

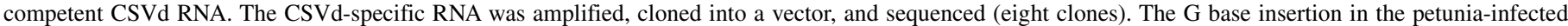

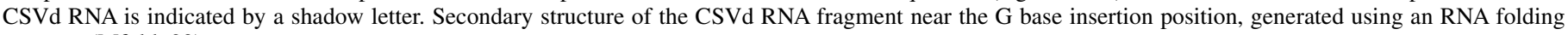
program (Mfold; 29). 
(Figs. 4 and 5), and could potentially create binding sites for host proteins to establish replication competence and to facilitate the mobility of the CSVd in these plants. However, the roles of these base substitutions in these CSVd isolates need to be studied further. Nevertheless, these studies clearly suggest that tomato plants may serve as a reservoir for CSVd replication and may help in transmitting CSVd infection to a wide range of plants, through either sap, seed, or pollen. CSVd disease was previously shown to be transmitted by tomato plants through both seed and pollen (12).

Similarly, it was reported that the PSTVd isolate KF 440-2 is infectious to potato and tomato plants upon mechanical inoculation, but not to Nicotiana tabacum plants. However, PSTVd-NT, which has a single base substitution (C259 $\rightarrow$ U259 substitution), was converted from the noninfectious PSTVd to an infectious RNA that is stable in both hosts (tomato and tobacco) (27). In the present studies, we observed that CSVd strain X16408 and CSVd-Petu appeared to be specific infectious agents in Ageratum and petunia, respectively. It is tempting to speculate that the widespread CSVd infection in chrysanthemum plants could be due to the remarkable ability of the CSVd RNA to replicate in commonly cultivated plants, such as tomato, potato, capsicum, petunia, and cucumber, as reported (1). Thus, many different types of plants serve as a reservoir for the CSVd strains, and they may contribute significantly to spreading the CSVd disease in chrysanthemum plants in the field.

In summary, in the present study, we analyzed a CSVd strain that frequently infects chrysanthemum plants in Japan. Our study suggested, for the first time, that linear CSVd (+) RNA replicates and produces disease symptoms in cultivated chrysanthemum plants as efficiently as the intact CSVd RNA isolated from the infected cultivated chrysanthemum plants. Moreover, the CSVd was found to replicate efficiently in a wide range of host plants. Thus, the frequent occurrence of CSVd infection in chrysanthemum cultivation appears primarily to result from the ability of CSVd to be transmitted from these additional host plants to chrysanthemum plants, in addition to transmission between chrysanthemum plants.
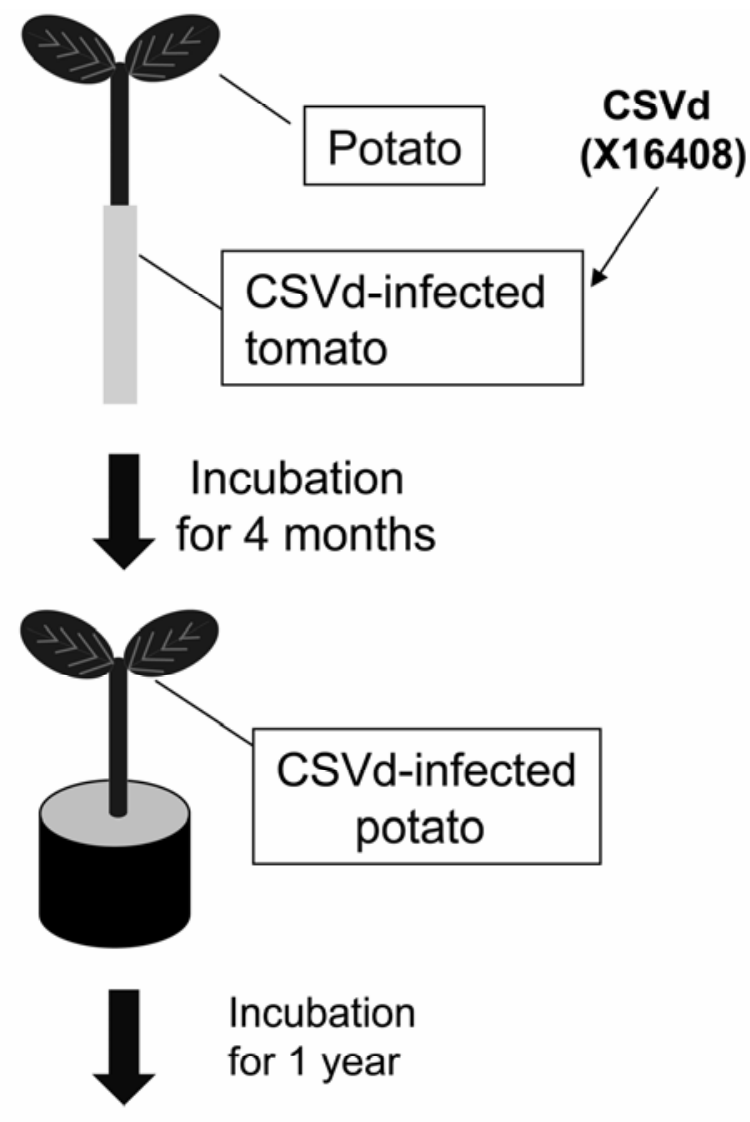

\section{RT-PCR and Sequencing}
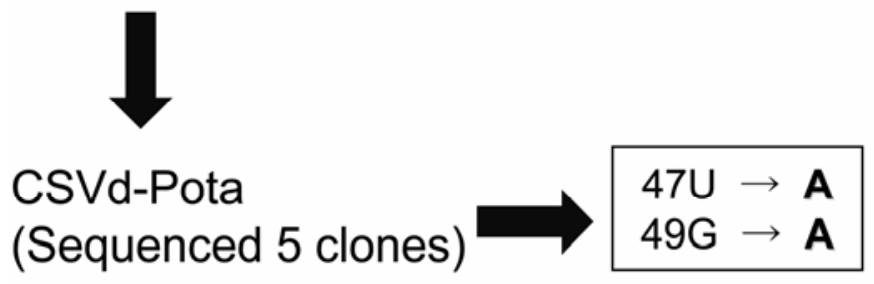

\section{Predicted of secondary structure of CSVd near the mutation sites}

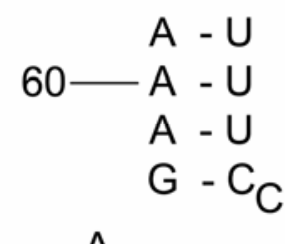

A

A

A

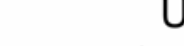

$A-U$

A - U

G - C

A $-U$

$A-U$

$50-\frac{}{G} A-U$

$U$

U

$U-A$

C - G

$G-C$

$U-A$

C C

G - C

$40-U-G$

CSV-X16408

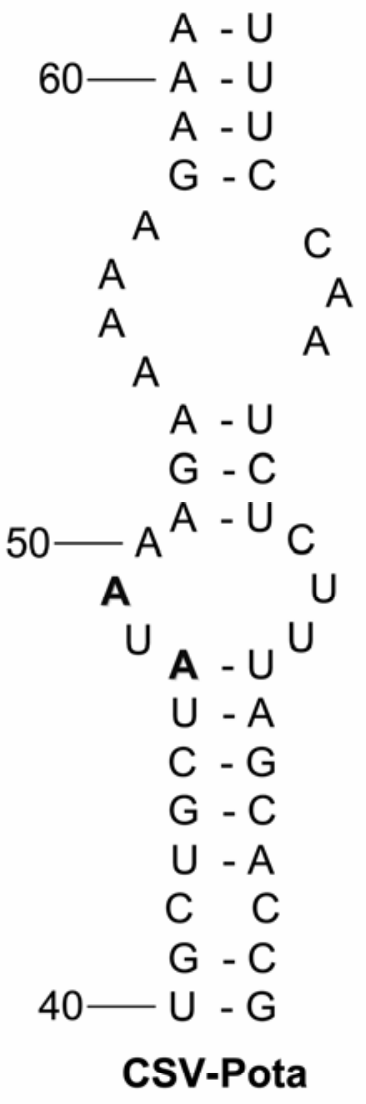

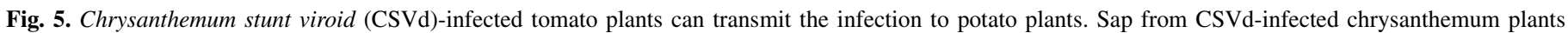

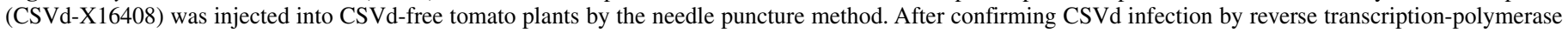

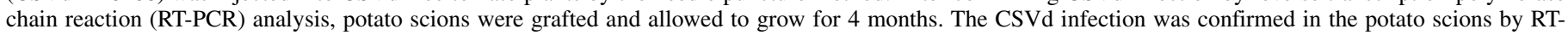

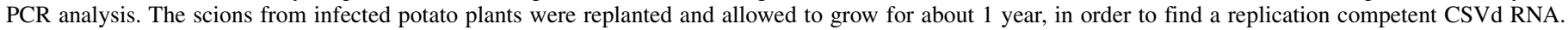

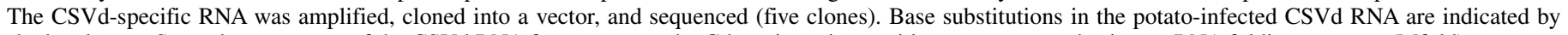
shadow letters. Secondary structure of the CSVd RNA fragment near the G base insertion position was generated using an RNA folding program (Mfold). 


\section{ACKNOWLEDGMENTS}

This work was supported by funds from the National Institute of Floricultural Science and the National Institute of Advanced Industrial Science and Technology (AIST) to Y. Matsushita and K. K. R. Penmetcha, respectively. We thank Y. Chikuo, T. S. Misono, and Y. Ito for their help and encouragement during this study.

\section{LITERATURE CITED}

1. Bouwen, I., and van Zaayen, A. 2004. Chrysanthemum stunt viroid. Page 219 in: Viroids. CSIRO, Melbourne.

2. Cress, D. E., Kiefer, M. C., and Owens, R. A. 1983. Construction of infectious potato spindle tuber viroid cDNA clones. Nucleic Acids Res. 11:6821-6835.

3. Diener, T. O. 1971. Potato spindle tuber "virus." IV. Replicating, low molecular weight RNA. Virology 45:411-428.

4. Diener, T. O. 1972. Potato spindle tuber viroid. VIII. Correlation of infectivity with a UV-absorbing component and thermal denaturation properties of the RNA. Virology 50:606-609.

5. Flores, R., Hernandez, C., Martinez de Alba, A. E., Daros, J. A., and DiSerio, F. 2005. Viroids and viroid-host interactions. Annu. Rev. Phytopathol. 43:117-139.

6. Gopinath, S. C. B., Matsugami, A., Katahira, M., and Kumar, P. K. R. 2005. Human vault-associated non-coding RNAs bind to mitoxantrone, a chemotherapeutic compound. Nucleic Acids Res. 33:4874-4881.

7. Gopinath, S. C. B., Misono, T. S., Kawasaki, K., Mizuno, T., Imai, M., Odagiri, T., and Kumar, P. K. R. 2006. An RNA aptamer that distinguishes between closely related human influenza viruses and inhibits haemagglutinin-mediated membrane fusion. J. Gen. Virol. 87:479-487.

8. Gross, H. J., Liebl, U., Alberty, H., Krupp, G., Domdey, H., Ramm, K., and Sanger, H. L. 1981. A severe and a mild potato spindle tuber viroid isolate differ in three nucleotide exchanges only. Bioscience Rep. 1:235241.

9. Haseloff, J., and Symons, H. R. 1981. Chrysanthemum stunt viroid: Primary sequence and secondary structure. Nucleic Acids Res. 9:27412752.

10. Horst, R. K., Langhans, R. W., and Smith, S. H. 1977. Effects of chrysanthemum stunt, chlorotic mottle, aspermy and mosaic on flowering and rotting of chrysanthemums. Phytopathology 67:9-14.

11. Keese, P., and Symons, R. H. 1985. Domains in viroids: Evidence of intermolecular RNA arrangements and their contribution to viroid evolution. Proc. Natl. Acad. Sci. USA 82:4582-4586.

12. Kryczyński, S., Paduch-Cichal, E., and Skrzeczkowski, L. J. 1988. Transmission of three viroids through seed and pollen of tomato plants. J. Phytopathol. 121:51-57.

13. Matsushita, Y., Tsukiboshi, T., Ito, Y., and Chikuo, Y. 2007. Nucleotide sequences and distribution of Chrysanthemum stunt viroid in Japan. J.
Jpn. Soc. Hort. Sci. 76:333-337.

14. Niblett, C. L., Dickson, E., Horst, R. K., and Romaine, C. P. 1979. Additional hosts and an efficient purification procedure for four viroids. Phytopathology 70:610-615.

15. Owens, R. A., Thompson, S. M., and Kramer, M. 2003. Identification of neutral mutants surrounding two naturally occurring variants of Potato spindle tuber viroid. J. Gen. Virol. 84:751-756.

16. Palukaitis, P., and Symons, R. H. 1980. Purification and characterization of the circular and linear forms of Chrysanthemum stunt viroid. J. Gen. Virol. 46:477-489.

17. Qi, Y., and Ding, B. 2003. Inhibition of cell growth and shoot development by a specific nucleotide sequence in a non-coding viroid RNA. Plant Cell 15:1360-1374.

18. Rakowski, A. G., and Symons, R. H. 1994. Infectivity of linear monomeric transcripts of citrus exocortis viroid: Terminal sequence requirements for processing. Virology 203:328-335.

19. Roberston, H. D., and Rosen, D. L., and Branch, A. D. 1985. Cell-free synthesis and processing of an infectious dimeric transcript of potato spindle tuber viroid RNA. Virology 142:441-447.

20. Rigden, J. E., and Rezaian, M. A. 1992. In vitro synthesis of an infectious viroid: Analysis of the infectivity of monomeric linear CEV. Virology 186:201-206.

21. Sano, T., Candresse, T., Hammond, R. W., Diener, T. O., Owens, R. A. 1992. Identification of multiple structural domains regulating viroid pathogenicity. Proc. Natl. Acad. Sci. USA 89:10104-10108.

22. Semancik, J. S. 1986. Separation for viroid RNAs by cellulose chromatography indicating conformational distinctions. Virology 155:39-45.

23. Tabler, M., and Sanger, H. L. 1984. Cloned single-and double-stranded DNA copies of potato spindle tuber viroid (PSTV) RNA and co-inoculated subgenomic DNA fragments are infectious. EMBO J. 3:3055-3062.

24. Tabler, M., and Sanger, H. L. 1985. Infectivity studies on different potato spindle tuber viroid (PSTV) RNA synthesized in vitro with SP6 transcription system. EMBO J. 4:2191-2199.

25. Verhoeven, J. T. J., Arts, M. S. J., Owens, R. A., and Roenhorst, J. W. 1998. Natural infection of petunia by chrysanthemum stunt viroid. Eur. J. Plant Pathol. 104:383-386.

26. Visvader, J. E., Forster, A. C., and Symons, R. H. 1985. Infectivity and in vitro mutagenesis of monomeric cDNA clones of citrus exocortis viroid indicates the site of processing of viroid precursors. Nucleic Acids Res. 13:5843-5866.

27. Wassenegger, M., Spieker, R. L., Thalmeir, S., Gast, F.-U., Riedel, L., Sanger, H. L. 1996. A single nucleotide substitution converts potato spindle tuber viroid (PSTVd) from a noninfectious to an infectious RNA for Nicotiana tabacum. Virology 226:191-197.

28. Zhong, X., Tao, X., Stombaugh, J., Leontis, N., and Ding, B. 2007. Tertiary structure and function of an RNA motif required for plant vascular entry to initiate systemic trafficking. EMBO J. 26:3836-3846.

29. Zuker, M. 2003. Mfold web server for nucleic acid folding and hybridization prediction. Nucleic Acids Res. 31:3406-3415. 